\title{
Estimación De Los Factores De Penalización Del Tiempo En La Función De Costo Generalizado En Bucaramanga Y Su Área Metropolitana
}

\section{Estimation Of Factors Of Penalty Time In The Function Of Generalized Cost In Bucaramanga And Its Metropolitan Area}

\author{
H Porras ${ }^{1}$, S Cote $^{2}$ \\ ${ }^{1} \mathrm{PhD}$. en Ingeniería Telemática. Docente e Investigador. UIS. Bucaramanga .Colombia.: hporras@ uis.edu.co \\ ${ }^{2}$ Ingeniera civil. Candidata a Magíster en Ingeniería Civil. UIS.Bucaramanga.Colombia.: sandra.cote@gmail.com
}

Recibido: jun 14 2016. Aceptado: jul 2, 2016. Versión final: jul 15, 2016

\begin{abstract}
RESUMEN
El tiempo de viaje es una medida natural de la operatividad de una vía o de cualquier sistema de transporte, en especial en sistemas masivos exclusivos tipo BRT (Bus Rapid Transit) o colectivo, donde uno de los principales objetivos del sistema de transporte público es el de movilizar personas, cargas, bienes y servicios. La medida o factor por el cual las personas miden subjetivamente la efectividad de la vía o del sistema de transporte, es el tiempo que les toma realizar el viaje, algunos elementos tales como el paisaje, el ambiente, la disponibilidad de asientos entre otros son elementos encargados de proporcionar una sensación de confort y ayudan a que la percepción del usuario con respecto al viaje sea mejor; las rutas simples también aportan sensación de confort al viaje realizado por el pasajero, pero su principal objetivo es llegar en el menor tiempo posible a su destino.

Cuando una persona realiza un viaje, el factor que más se revisa es el tiempo de viaje, pero también al realizar la programación de rutas de transporte público se debe incorporar atributos cualitativos al viaje tales como confort, seguridad y prestigio del modo de transporte. Es decir el valor del tiempo suele ser mayor en situaciones de congestión o donde el viajero no se encuentra confortable, Ortúzar sugiere que el costo del tiempo de viaje debe ser medido por segmento de viaje. Algunos de los tiempos que se consideran son: El tiempo de caminata hasta el paradero, el tiempo de espera en el paradero, el tiempo de viaje en el bus (En este aspecto se debe considerar si el bus está desocupado o no, esto con el fin de revisar el confort y la comodidad en el viaje).
\end{abstract}

Palabras clave: Encuestas de preferencia declarada, Función de costo generalizado, Modelo de elección discreta, Modelación de la demanda, Planeación del transporte

\begin{abstract}
The travel time is a natural measure of the operation of a route or a transport system, where one of the main objectives is to transport people, cargo, goods and services. The measure or factor by which people measure subjectively the effectiveness of the road or transportation system is the time it takes to travel, some elements such as the landscape, the environment, the availability of seats and others are elements responsible for providing a sense of comfort and help the user perception regarding the trip better, simpler routes also provide comfort feeling trip by the passenger, but its main goal is to reach as quickly as possible to your destiny.

When a person takes a trip, the factor that is reviewed is the travel time, but also to make the programming of public transport routes should be incorporated to travel qualitative attributes such as comfort, safety and prestige of the transport mode. That is the time value is usually higher in situations of congestion or where the traveler is not comfortable, so the author in his book Ortúzar Transportation Demand Models suggests that the cost of travel time
\end{abstract}

Este artículo puede compartirse bajo la licencia CC BY-ND 4.0 (https://creativecommons.org/licenses/by-nd/4.0/). H Porras, S Cote. "Estimación De Los Factores De Penalización Del Tiempo En La Función De Costo Generalizado En Bucaramanga Y Su Área Metropolitana”, UIS Ingenierías, vol. 15, no. 2, pp. 135-144, jul-dic 2016, doi: http://dx.doi.org/10.18273/revuin.v15n2-2016012 
should be measured by travel segment. Some of the times that are considered are: the walking time to the whereabouts, the waiting time at the bus stop and the travel time on the bus (in this aspect should be considered if the bus is idle or not, this in to review the accommodations on the trip).

Keywords: Stated preference surveys, Generalized cost function, Discrete choice model, Demand modeling, transportation planning.

\section{INTRODUCCIÓN}

Este documento, se centra en estimar los factores de penalización del tiempo en la función del costo generalizado para el transporte público. Para ello es importante especificar dicha ecuación. Esta se puede definir como la "ecuación que incluye el tiempo de caminata, el tiempo de espera, el tiempo en vehículo y los tiempos de transferencia y el costo de la tarifa, afectada por unos factores de penalización" [1], y se emplea en la mayoría de métodos de asignación de demanda de viajes dado que incluye componentes tales como tiempo de viaje y tarifas, se podría afirmar que estos elementos son los que determinan la satisfacción del usuario. De acuerdo a Ortúzar, señala que "para la obtención de los factores de ponderación de los tiempos caminata, espera y trasbordo, usualmente se utilizan métodos de encuestas de preferencias reveladas o declaradas" [1]. Para ello es necesario además de definir la ecuación de costo generalizado del tiempo, diferenciar las encuestas de preferencia declarada, de preferencia revelada, o modelos en encuesta mixta donde se incluyen encuestas de Preferencia Declarada y Preferencia Revelada dado que los tiempos a los que se hace referencia en la ecuación provienen de la percepción que tienen los usuarios de sus tiempos de traslado. Desde el punto de vista de la planificación del transporte, es necesario predecir los flujos que se presentarán en el sistema para diferentes situaciones, es decir predecir el usuario al cual se atiende con el sistema de transporte, cuál es su punto de partida

Ahora bien "desde el punto de vista de transporte el desarrollo del sistema masivo, se basa en la implementación de un modelo de asignación de transporte, a través del cual se estimó la demanda potencial del nuevo sistema de transporte, para ello se centra en la elección de modos y se fundamenta en el ahorro del tiempo de viaje, que se calcula como el tiempo total que emplean los viajeros para desde el inicio hasta el fin del viaje; la ponderación de estos componentes del tiempo de viaje y costo monetario se expresa matemáticamente para el algoritmo del modelo como una suma polinómica.

A este valor se le denomina en ingeniería de transporte como el costo generalizado del viaje, estos factores se obtienen a partir de las encuestas de preferencias declaradas, las cuales se plantean como la base para la estimación de la demanda" [2]. La ecuación por medio y cual su punto de llegada, para esto, es necesario conocer el comportamiento humano y de esta forma poder hacer una aproximación en la demanda de transporte.

En la actualidad, es posible predecir el comportamiento humano en respuesta a cambios en el sistema de transporte de un individuo o de un grupo de individuos ante situaciones cambiantes del sistema. Las decisiones de las personas sobre los viajes que deben efectuar como parte de sus actividades cotidianas conducen directamente a una "demanda" o un "deseo" de viaje. Por su naturaleza dinámica, la demanda responde también a cambios en los niveles de servicio, a tal grado que se puede presentar una competencia permanente entre los diversos medios de transporte disponibles en una ciudad.

\section{METODOLOGÍA}

El constante interés del ser humano por optimizar la movilidad, mejorando los sistemas de transporte público, enfocado especialmente en la disminución de los tiempos que debe permanecer viajando ha impulsado el desarrollo de estudios sobre el tiempo, analizándolo según las etapas en que este puede dividirse, por ejemplo: tiempo que debe caminar para abordar, el que debe permanecer a bordo del medio de transporte que elija, el tiempo de espera y se debe incluir un tiempo adicional cuando se realiza uno o más trasbordos, para finalmente llegar al destino.

de la cual se incluyen estos factores de penalización se define como la función del costo generalizado, esta incluye el tiempo de caminata, el tiempo de espera, el tiempo en vehículo y los tiempos de transferencia y el costo de la tarifa, afectada por unos factores de llamados factores de penalización, esta función escrita de forma general:

$$
\begin{array}{r}
C_{k}=\sum_{i e j}\left[r_{j}+\operatorname{VOT} *\left(\gamma_{\chi} \chi_{j}+\gamma_{\omega} \omega_{j}\right)\right]+\sum_{i \in I}[\operatorname{VOT} * \\
\left.\left(\gamma_{d} d_{i}+\gamma_{v} t_{i}\left(1+\alpha\left(v i / C_{i}\right) \beta\right)\right)\right]
\end{array}
$$

Dónde:

$C_{k}=$ Costo total del viaje $K$ en unidades monetarias

$C_{i}=$ Capacidad ofrecida por cada ruta para el periodo

de estudio

$d_{i}=$ Tiempo en paradero 
$i=$ Índice de los arcos a través de los cuales se realiza el viaje $K$

$l=$ Selección de arcos usados en el viaje $K$

$j=$ Índice de las rutas usadas en el viaje $K$

$J=$ Selección de rutas usadas en el viaje $K$

$r_{j}=$ Tarifa para el conjunto de rutas usadas en el viaje

$t_{i}=$ Tiempo de viaje a bordo del vehículo o tiempo caminando en el arco $i$

$v_{i}=$ Volumen de pasajeros en el arco

VOT $=$ Valor del tiempo

$\omega i=$ Tiempo de espera para abordar la ruta

$\chi_{j}=$ Tiempo de penalización al transferir de una prueba a otra

$\gamma_{d}=$ Factor multiplicador que penaliza el tiempo en paradero

$\gamma_{v}=$ Factor multiplicador que penaliza el tiempo a bordo del vehículo

$\gamma_{\omega}=$ Factor multiplicador que penaliza el tiempo de espera

$\gamma_{\chi}=$ Factor multiplicador que penaliza el tiempo de transferencia

$\alpha, \beta=$ Parámetros de congestión" [3]

Dentro de la función de costo generalizado, las variables definidas como $\gamma_{d}, \gamma_{v}, \gamma_{w} y \gamma_{x}$, son los factores de ponderación de los tiempos adicionales incluidos en la ecuación de costo generalizado, es decir, el tiempo total que gasta un usuario desde que inicia su viaje hasta que llega a su destino, donde el tiempo de caminata, de espera, de transferencia y de parada, son penalizados (aumentados), dado que se traducen en molestia para los usuarios [4].

De acuerdo al nivel y las características socioeconómicas que presente la persona, elegirá de acuerdo a las alternativas la que según su criterio sea mejor. Para incorporar la mejor opción para el usuario, se suele utilizar el concepto de utilidad, el cual se define como lo que el individuo desea maximizar. Utilidad, representa la satisfacción de un individuo cuando gasta sus recursos sobre diferentes cosas.

"La construcción de utilidad más común es un modelo lineal, en el cual la combinación de los atributos es aditiva:

$$
\mathrm{U}_{\mathrm{i}}=\mathrm{a}_{0}+\mathrm{a}_{1} \mathrm{X}_{1}+\mathrm{a}_{2} \mathrm{X}_{2}+\ldots \mathrm{a}_{\mathrm{n}} \mathrm{X}_{\mathrm{n}}
$$

Dónde:

$$
\begin{aligned}
& U_{i}=\text { Utilidad de opción } i \\
& X_{i} \ldots X_{n}=\text { Productos de atributos } \\
& a_{i} \ldots a_{n}=\text { Modelos de Coeficientes } \\
& a_{0}=\text { Modelos de Constantes }
\end{aligned}
$$

La utilidad se define como una combinación lineal de variables, en la que cada variable representa una característica de la alternativa, lo que ayuda a distinguirla de las demás. La influencia relativa de cada atributo ésta dada por su coeficiente. La constante modal específica $\mathrm{a}_{0}$, se interpreta como la representación de la influencia neta de todas aquellas características no observadas o no incluidas en forma explícita en la utilidad de cada alternativa" [3].

"Para la obtención de los factores de ponderación de los tiempos caminata, espera y trasbordo, usualmente se utilizan métodos de encuestas de preferencias reveladas o declaradas. La función de utilidad en transporte público elaborada a través de la aplicación de encuestas de preferencia declarada es:

$$
\begin{gathered}
\mathrm{U}=\text { cte }+\alpha \cdot \mathrm{T}_{\text {caminata }}+\beta \cdot \mathrm{T}_{\text {espera }}+\lambda \cdot \mathrm{T}_{\mathrm{vehiculo}}+ \\
\varphi \cdot \mathrm{T}_{\text {trasbordo }}+\theta \cdot \text { Tarifa }(3)[5]
\end{gathered}
$$

Dónde:

$U=$ función de utilidad en transporte público.

Cte. $=$ Constante .

Tcam = Tiempo de caminata (tanto en el origen, destino)

Tesp = Tiempo de espera en el paradero del bus.

Tveh = Tiempo de viaje dentro del vehículo.

Ttrasb $=$ Trasbordo.

Tarifa $=$ Valor que se paga por el servicio.

$\theta \varphi \lambda \beta \alpha,,=$ Factores de penalización a estimarse."

\subsection{ENCUESTAS DE PREFERENCIA DECLARADA}

Es necesario además de definir la ecuación de costo generalizado del tiempo, conocer las encuestas de preferencia declarada, dado que los tiempos a los que se hace referencia en la ecuación provienen de la percepción que tienen los usuarios de sus tiempos de traslado.

Desde el punto de vista de la planificación del transporte, es necesario predecir los flujos que se presentarán en el sistema para diferentes situaciones. Las decisiones de las personas sobre los viajes que deben efectuar como parte de sus actividades cotidianas conducen directamente a una "demanda" o un "deseo" de viajes. Por su naturaleza dinámica, la demanda responde también a cambios en los niveles de servicio, a tal grado que se puede presentar una competencia permanente entre los diversos medios de transporte disponibles en una ciudad.

Como se ha mencionado anteriormente para la actualización de estos factores es preciso emplear encuestas de preferencia declarada (PD) o encuestas de preferencia revelada (PR) o utilizar los dos tipos de encuesta. El término encuestas de Preferencia Revelada se relacionan con la intención de modelar el mundo como 
es [6], en este tipo de encuesta se revisa las alternativas elegidas y no elegidas por el individuo las cuales permiten estimar con tecnicas estadistica, la función utilidad de la alternativa que represente las preferencias de cada individuo, tiene algunos inconvenientes tales como: No permite hacer estimación de modelos adecuados dado que se centra solo en la realidad, presenta dificultad para evaluar el impacto de variable cualitativas como comodidad, fiabilidad, seguridad, confort, entre otras, además en este tipo de encuestas no es posible analizar la demanda de alternativas no existentes en el mercado.

En cuanto a las encuestas de Preferencia Declarada, se relacionan con el deseo de modelar como sería el mundo, en este tipo de encuesta el investigador plantea una serie de situaciones o escenarios ficticios a partir de los cuales desea modelar una situación y el individuo selecciona de las opciones las de su preferencia, a diferencia de las PR las PD pueden construir escenarios hipoteticos, puede aislar efectos de otras variables, el individuo debe considerar entre 8 o 9 escenarios, los cuales generan multiples respuestas[4].

Algunos autores recomiendan el uso de los dos tipos de encuesta dado que las PR representaria las preferencias que toma el individuo ante lo que actualmente esta ocurriendo y las PD muestran el comportamiento del individuo ante diferentes escenarios [7].

Una vez realizada las encuestas se realiza el proceso de validación y tratamiento de los datos, para esto existen diversas formas de estimar los parametros de las funciones de utilidad, entre ellos se encuentran: Técnicas de Regresión, MANANOVA (Monotonic analisis of variance), y el Utilización de metodos de elección discreta.

La utilización de Metodos de elección discreta, corresponde a técnicas estadisticas mas avanzadas aplicadas al análisis de datos de PD. Son muy utilizados por su robustez y aplicabilidad tanto a elecciones como escalonamientos y jerarquizaciones a través de transformaciones apropiadas. Se basa en el principio de maximización de la verosimilitud.[2]. Al modelar datos con PD se tiene garantia que las variables explicativas son exactas, dada la naturaleza de los experimentos, pero también se le atribuye a estos modelos es la potencial ausencia de compromiso entre lo que el individuo declara y lo que realmente va a hacer [6]

Es importante aclarar que estos factores de penalización al provenir de encuestas PD, son muy subjetivos, dado que dependen como se mencionó anteriormente de la percepción que tenga el individuo sobre su viaje, algunos factores que pueden afectar el tiempo de viaje: Hora en la cual se realiza el viaje, si hay congestión, demora, retrasos o si esta normal, propósito del viaje, longitud y ahorro del tiempo de viaje, además de atributos tales como confort, seguridad, paisaje, comodidad entre otros.

Para definir la población a la cual se le realizará un muestreo aleatorio estratificado teniendo en cuenta la ubicación geográfica y socio - económica (estratos) de los habitantes del área metropolitana de Bucaramanga (Bucaramanga, Girón, Floridablanca y Piedecuesta) Una vez definida la población se debe realizar el proceso selección de la muestra. Para el desarrollo de este estudio se utilizará el muestreo tradicional, ya que este permite seleccionar toda la muestra la cual es aleatoria antes de iniciar la recopilación de datos. Para realizar la selección del tamaño de la muestra se utilizó la información estadística que se encontraba en la página web del Departamento Administrativo Nacional de estadística (DANE) población del área metropolitana de Bucaramanga proyectada al año 2013, dado que este año se realizará el muestreo de campo, a continuación en la siguiente Tabla:

TABLA I.

POBLACIÓN DANE 2013 PARA BUCARAMANGA Y SU ÁREA METROPOLITANA

\begin{tabular}{|l|r|}
\hline & Población \\
\hline Bucaramanga & 526.940 \\
\hline Floridablanca & 263.951 \\
\hline Girón & 170.706 \\
\hline Piedecuesta & 142.448 \\
\hline Total & 1.104 .045 \\
\hline
\end{tabular}

Fuente: Dane

Para el cálculo del tamaño de la muestra se hará en base a la siguiente fórmula:

$$
N=\frac{p^{*} q}{\frac{e^{2}}{z^{2} / 2}}
$$

Dónde:

$p=$ Probabilidad de éxito

$q=$ Definida como $(1-p)$

$e=$ Error máximo permitido

$\mathrm{Z}=$ Valor distribución normal correspondiente a la probabilidad del error

Para el estudio se utilizará una $\mathrm{p}=0,5$ ya que es lo más recomendable para garantizar una muestra significativa y representativa de la población total, para los otros valores tenemos un $\mathrm{e}=0.05$ y un $\mathrm{Z}=1.96$ obtenido de acuerdo al error. 
Reemplazando los valores en la fórmula obtenemos una muestra de 383.16 o sea: 385 ENCUESTAS.

Al realizar una desagregación en términos de población por estrato a partir de la encuesta de hogares por estrato, información tomada por el DANE, donde se obtuvo la siguiente tabla:

TABLA II

HOGARES POR ESTRATO PARA BUCARAMANGA Y SU ÁREA METROPOLITANA

\begin{tabular}{|c|c|c|c|c|c|c|c|}
\hline & Estrato 1 & Estrato 2 & Estrato 3 & Estrato 4 & Estrato 5 & Estrato 6 & total \\
\hline Bucaramanga & 8057 & 22253 & 27219 & 32419 & 3161 & 6112 & 99221 \\
\hline Floridablanca & 3037 & 17003 & 17054 & 6386 & 1870 & 379 & 45729 \\
\hline Giron & 4409 & 7837 & 8013 & 553 & 44 & 3 & 20859 \\
\hline Piedecuesta & 287 & 4858 & 11547 & 935 & 41 & 15 & 17683 \\
\hline
\end{tabular}

Fuente: Dane

A partir de la anterior tabla se realizó una distribución porcentual para identificar como se distribuía la cantidad de encuestas a realizar se obtuvo la siguiente información:

TABLA III.

ENCUESTAS APLICADAS PARA BUCARAMANGA Y SU ÁREA METROPOLITANA

\begin{tabular}{|c|c|c|c|c|c|c|}
\hline & Estrato 1 & Estrato 2 & Estrato 3 & Estrato 4 & Estrat0 5 & total \\
\hline Bucaramanga & 17 & 47 & 58 & 69 & 20 & 210 \\
\hline Floridablanca & 6 & 35 & 35 & 13 & 5 & 94 \\
\hline Giron & 9 & 16 & 17 & 1 & 0 & 43 \\
\hline Piedecuesta & 1 & 10 & 25 & 2 & 0 & 38 \\
\hline
\end{tabular}

Fuente: Elaboración Propia

Estas encuestas se realizaron durante varios días, dentro de semanas normales y se procuró encuestar tanto a hombres como mujeres.

\section{RESULTADOS}

Para el paso siguiente en el análisis y obtención de los resultados se utilizó la metodología plateada por Sartori, donde se menciona que es necesario plantear este tipo de encuestas para realizar un correcto análisis de los viajes a bordo de cualquier sistema de transporte, según plantea el autor de esta forma se obtienen resultados más coherentes con la realidad de elección de los individuos. Ahora bien de acuerdo al autor "El formulario aplicado contenía un grupo de preguntas con preferencias reveladas sobre la duración y etapas del viaje realizado por el usuario, a lo largo del formulario se le pregunto 3 veces el tiempo de viaje empleado entre el origen y destino: Primero se le indagó por la hora de inicio y la hora de finalización del viaje, después se le pregunto puntualmente por la duración estimada del viaje y finalmente se indagó por el tiempo o cuadras que gastaba en las diferentes etapas del viaje: Caminata, espera y en vehículo" [7], A continuación en la Tabla 3, tendremos el resultado de estos tiempos:

TABLA IV

TIEMPO PROMEDIO DE VIAJE POR ESTRATO SOCIOECONÓMICO

\begin{tabular}{|c|c|c|c|}
\hline ESTRATO & $\begin{array}{c}\text { TIEMPO TOTAL } \\
\text { Inicio - Fin }\end{array}$ & $\begin{array}{c}\text { Tiempo Total } \\
\text { Reportado }\end{array}$ & $\begin{array}{c}\text { Tiempo } \\
\text { Estimado por } \\
\text { Etapas }\end{array}$ \\
\hline 1 & 73,87 & 74,97 & 76,73 \\
\hline 2 & 67,39 & 69,75 & 71,24 \\
\hline 3 & 63,26 & 62,18 & 64,73 \\
\hline 4 & 59,47 & 60,35 & 62,12 \\
\hline 5 & 61,16 & 60,29 & 63,37 \\
\hline
\end{tabular}

Fuente: Elaboración Propia

Como se aprecia en la tabla 5 a partir de la percepción que tiene el usuario, cuando este toma el escenario de tomar un alimentador (Para nuestra consideración llamamos alimentador tanto al bus convencional como las rutas alimentadoras del SITM Metrolinea) camina en promedio 1,5 cuadras mientras que para los otros dos escenarios debe caminar aproximadamente 3 cuadras. En el escenario de destino o llegada se puede afirmar que camina en promedio 2 cuadras para cualquiera de los escenarios.

Tal como se planteó y siguiendo los parámetros del autor Sarmiento en su publicación, donde sugiere que para la selección del modelo para generar la ecuación del costo generalizado del tiempo emplea la función logit, dado que esta permite predecir la probabilidad que ocurra o no algún evento en función de un numero de factores explicativos o independientes.

Como se indicó, se empleará la siguiente función de utilidad a emplear dentro del experimento, esta función corresponde a la función de costo generalizado para el transporte:

$\mathrm{U}=$ cte $+\alpha \cdot \mathrm{T}_{\text {caminata }}+\beta \cdot \mathrm{T}_{\text {espera }}+\lambda \cdot \mathrm{T}_{\text {vehiculo }}+$

$\varphi \cdot \mathrm{T}_{\text {trasbordo }}+\theta \cdot$ Tarifa 
TABLA V

TIEMPO PROMEDIO DE VIAJE EN MINUTOS POR TIPO DE TRANSBORDO Y ESTRATO SOCIOECONÓMICO

\begin{tabular}{|c|c|c|c|c|c|c|c|}
\hline TRANSBORDO & ESTRATO & $\begin{array}{l}\text { Promedio Cuadras } \\
\text { Caminadas al Inicio }\end{array}$ & $\begin{array}{l}\text { Promedio } \\
\text { Tiempo Espera } \\
\text { 1er Vehiculo }\end{array}$ & $\begin{array}{c}\text { Promedio } \\
\text { Tiempo dentro } \\
\text { 1erVehiculo }\end{array}$ & $\begin{array}{c}\text { Promedio } \\
\text { Tiempo Espera } \\
\text { 2do Vehiculo }\end{array}$ & $\begin{array}{c}\text { Promedio } \\
\text { Tiempo dentro } \\
\text { 2do Vehiculo }\end{array}$ & $\begin{array}{c}\text { Promedio Cuadras } \\
\text { Caminadas al } \\
\text { destino }\end{array}$ \\
\hline \multirow{3}{*}{$\begin{array}{c}\text { TRONCAL + } \\
\text { ALIMENTADOR }\end{array}$} & 3 & 3,2 & 7,6 & 37,1 & 10,27 & 12,16 & 2,16 \\
\hline & 4 & 3 & 8 & 32,9 & 16,19 & 14,42 & 1,57 \\
\hline & 5 & 2 & 7,3 & 27,2 & 13,1 & 11,1 & 2,41 \\
\hline Promedio & & 2,7 & 7,6 & 32,4 & 13,2 & 12,6 & 2,05 \\
\hline \multirow{5}{*}{$\begin{array}{l}\text { ALIMENTADOR + } \\
\text { TRONCAL }\end{array}$} & 1 & 6,3 & 10 & 8,2 & 12,25 & 22,08 & 2 \\
\hline & 2 & 4,2 & 8,4 & 10 & 10,12 & 21,06 & 1,32 \\
\hline & 3 & 1,5 & 6,4 & 12,5 & 9,32 & 28,04 & 2 \\
\hline & 4 & 1 & 5,2 & 6 & 10,16 & 31,47 & 2,3 \\
\hline & 5 & 2,5 & 4 & 15 & 12,21 & 27,03 & 2,6 \\
\hline Promedio & & 3,1 & 6,8 & 10,34 & 10,81 & 25,936 & 2,044 \\
\hline $\begin{array}{l}\text { ALIMENTADOR + } \\
\text { ALIMENTADOR }\end{array}$ & 3 & 1,5 & 5,3 & 21,3 & 13,18 & 21,18 & 1,7 \\
\hline Promedio & & 1,5 & 5,3 & 21,3 & 13,18 & 21,18 & 1,7 \\
\hline
\end{tabular}

Fuente: Elaboración Propia

Dónde:

$\mathrm{U}=$ función de utilidad en transporte público.

Cte. $=$ Constante .

Tcam = Tiempo de caminata (tanto en el origen, destino)

Tesp = Tiempo de espera en el paradero del bus.

Tveh = Tiempo de viaje dentro del vehículo .

Ttrasb = Trasbordo

Tarifa = Valor que se paga por el servicio.

$\theta \quad \theta \quad \beta \alpha,,=$ Factores de penalización $a$ estimarse."

Ahora bien la probabilidad de elección de una alternativa está dada por:

$P_{1}=\frac{\mathrm{e}^{\psi \mathrm{U}_{1}}}{\mathrm{e}^{\psi \mathrm{U}_{1}}+\mathrm{e}^{\psi \mathrm{U}_{0}}}(5)$

Donde P1, implica la probabilidad de elegir la alternativa 1 respecto a la alternativa 0 , de acuerdo a la utilidad (U), percibida por el usuario considerando las variables mostradas en la función de utilidad.1

Para resolver este tipo de problemas se empleó el Software BIOGEME, este es un código abierto gratuito diseñado para la estimación de modelos de elección discreta. Para poder emplear este software se requiere dos tipos de archivo de input, uno tipo .mod, en este archivo es donde se definen las variables a emplear en el modelo, parámetros a estimar y la función utilidad, el segundo archivo se debe ingresar como un tipo DAT que es donde se coloca la información de las encuestas.

De acuerdo a Ortúzar en su libro de Modelos Econométricos plantea que es posible evaluar el nivel de

\footnotetext{
${ }^{1}$ Citado "Modelos de Demanda de Transporte (2000)[1] - Capítulo 4 - Modelos de Elección Discreta - Pg. 167-173”
}

confianza por medio de Test-t para la significancia de un parámetro $\phi$, es decir que como en nuestro caso para la selección de la muestra se tomó un nivel de confianza de $95 \%$, de acuerdo a la curva se tiene un valor de 1.96 para que las variables sean incluidas en el modelo.

Para generar un modelo de ecuación de costo generalizado de viaje que se ajuste a los datos obtenidos con las encuestas realizadas y para ello se establece una serie de escenarios que te permiten evaluar como es el ajuste de cada uno y para verificar dicho ajuste se emplea test de bondad de ajuste tipo t-student, para ello se plantean diferentes modelos y de estos seleccionar el más adecuado. Para ello se establecen diferentes escenarios realizando combinación de estratos.

TABLA VI.

MODELOS EVALUADOS

\begin{tabular}{|c|l|}
\hline Escenario & \multicolumn{1}{|c|}{ Descripción } \\
\hline 1 & Agrupa todos los estratos, sin considerar motivo del viaje y frecuencia \\
\hline 2 & Cada estrato por separado \\
\hline 3 & Se evalua estrato 1 y 2,3y4y 5 solo sin considerar motivo del viaje y frecuencia \\
\hline
\end{tabular}

Fuente: Elaboración Propia

Como apreciamos al evaluar el primer escenario, los valores obtenidos para el Test - t, algunos se encuentran por fuera del rango permitido para ser considerado el modelo de nuestra función. 
TABLA VII.

ESCENARIO1 EVALUADO

MODELO 1
\begin{tabular}{|l|c|c|c|c|}
\hline VARIABLE & PARAMETRO & ERROR ESTANDAR & T-TEST & FP Y VST \\
\hline CONSTANTE & $-0,917$ & 0,283 & $-2,17$ & \\
\hline TCAM & $-0,0397$ & 0,0386 & $-1,32$ & 1,36 \\
\hline TESP & $-0,0336$ & 0,0365 & $-0,87$ & 1,18 \\
\hline TVEH & $-0,0325$ & 0,0316 & $-1,99$ & 1 \\
\hline COSTO & $-0,00026$ & 0,00162 & $-0,26$ & 87,36 \\
\hline TRANSBORDO & $-0,0246$ & 0,132 & $-0,21$ & 0,81 \\
\hline Muestra & 1544 & & & \\
\cline { 4 - 6 }
\end{tabular}

Fuente: Elaboración Propia

Ahora se realiza la prueba para el segundo escenario, en el cual se considera cada estrato por separado:

TABLA VIII

ESCENARIO 2 ESTRATO 1 EVALUADO

\begin{tabular}{|c|c|c|c|c|}
\hline MODELO 2 & & & & \\
\hline \multicolumn{5}{|l|}{ ESTRATO 1} \\
\hline VARIABLE & PARAMETRO & ERROR ESTANDAR & T-TEST & FPYVST \\
\hline CONSTANTE & \begin{tabular}{|c|}
$-1,003$ \\
\end{tabular} & 1,16 & $-1,86$ & \\
\hline TCAM & $-0,119$ & 0,207 & $-1,77$ & 1,47 \\
\hline TESP & $-0,196$ & 0,193 & $-1,92$ & 1,75 \\
\hline TVEH & $-0,135$ & 0,182 & $-1,73$ & 1 \\
\hline COSTO & $-0,00227$ & 0,022 & $-1,27$ & 61,39 \\
\hline TRANSBORDO & $-1,029$ & 0,187 & -1 & 10,25 \\
\hline Muestra & 132 & & & \\
\hline
\end{tabular}

Fuente: Elaboración Propia

TABLA IX.

ESCENARIO 2 ESTRATO 2 EVALUADO

ESTRATO2
\begin{tabular}{|l|c|c|c|c|}
\hline VARIABLE & PARAMETRO & ERROR ESTANDAR & T-TEST & FP YVST \\
\hline CONSTANTE & 0,323 & 0,542 & $-1,27$ & \\
\hline TCAM & $-0,096$ & 0,085 & $-1,07$ & 1,62 \\
\hline TESP & $-0,0522$ & 0,081 & $-1,03$ & 1,27 \\
\hline TVEH & $-0,0503$ & 0,055 & $-1,93$ & 1 \\
\hline COSTO & $-0,00212$ & 0,002 & $-1,72$ & 41,19 \\
\hline TRANSBORDO & $-0,316$ & 0,263 & $-1,43$ & 8,42 \\
\hline Muestra & 436 & & & \\
\hline
\end{tabular}

Fuente: Elaboración Propia
TABLA X. ESCENARIO 2 ESTRATO 3 EVALUADO

\begin{tabular}{|c|c|c|c|c|}
\hline ESTRATO 3 & & & & \\
\hline VARIABLE & PARAMETRO & ERROR ESTANDAR & T-TEST & FP Y VST \\
\hline CONSTANTE & 0,246 & 0,621 & $-0,32$ & \\
\hline TCAM & $-0,0973$ & 0,063 & $-1,65$ & 1,97 \\
\hline TESP & $-0,0451$ & 0,059 & $-1,61$ & 1,75 \\
\hline TVEH & $-0,0218$ & 0,042 & $-1,16$ & 1 \\
\hline COSTO & $-0,00072$ & 0,002 & $-1,78$ & 59,32 \\
\hline TRANSBORDO & $-0,284$ & 0,201 & $-1,67$ & 8,01 \\
\hline Muestra & 536 & & & \\
\hline
\end{tabular}

Fuente: Elaboración Propia

TABLA XI

ESCENARIO 2 ESTRATO 4 EVALUADO

ESTRATO 4
\begin{tabular}{|l|c|c|c|c|}
\hline VARIABLE & PARAMETRO & ERROR ESTANDAR & T-TEST & FP YVST \\
\hline CONSTANTE & $-3,032$ & 1,152 & $-4,12$ & \\
\hline TCAM & $-0,176$ & 0,136 & $-3,16$ & 3,12 \\
\hline TESP & $-0,0116$ & 0,121 & $-2,17$ & 1,96 \\
\hline TVEH & $-0,0921$ & 0,096 & $-1,99$ & 1 \\
\hline COSTO & $-0,00096$ & 0,004 & $-2,65$ & 61,87 \\
\hline TRANSBORDO & 0,872 & 0,601 & $-2,01$ & 12,19 \\
\hline Muestra & 340 & & & \\
Fuente: Elaboración Propia
\end{tabular}

Fuente: Elaboración Propia

TABLA XII.

ESCENARIO 2 ESTRATO 5 EVALUADO

\begin{tabular}{|l|c|c|c|c|}
\hline ESTRATO 5 & \multicolumn{1}{|l|}{} \\
\hline VARIABLE & PARAMETRO ERROR ESTANDAR & T-TEST & FP YVST \\
\hline CONSTANTE & $-2,16$ & 2,631 & $-2,46$ & \\
\hline TCAM & $-0,115$ & 0,305 & $-2,53$ & 2,31 \\
\hline TESP & $-0,087$ & 0,287 & $-2,62$ & 1,95 \\
\hline TVEH & $-0,0362$ & 0,259 & $-2,78$ & 1 \\
\hline COSTO & $-0,00051$ & 0,01 & $-1,47$ & 63,16 \\
\hline TRANSBORDO & $-0,221$ & 0,986 & $-2,51$ & 3,12 \\
\hline Muestra & 100 & & & \\
\hline
\end{tabular}

Fuente: Elaboración Propia

En este caso los valores para Test $-\mathrm{t}$ se encuentran aún más dispersos y varios de estos no se encuentran en el rango permisible.

En el tercer modelo se contempla realizar una agrupación de los estratos, es así como tenemos Estrato 1 y 2, Estrato 3 y 4 , y Estrato 5. 


\begin{tabular}{|c|c|c|c|c|c|}
\hline ESTRATO & TCAM & TESP & TVEH & COSTO VST & T TRANSB \\
\hline 1 Y 2 & 1,25 & 1,37 & 1 & 19,83 & 7,35 \\
\hline 3 Y 4 & 1,89 & 1,56 & 1 & 43,93 & 5,16 \\
\hline 5 & 2,31 & 1,95 & 1 & 63,16 & 3,12 \\
\hline
\end{tabular}

TABLA XIII.

ESCENARIO 3 ESTRATO 1 Y 2 EVALUADO

ESTRATO 1Y 2
\begin{tabular}{|l|c|c|c|c|}
\hline VARIABLE & PARAMETRO & ERROR ESTANDAR & T-TEST & FP YVST \\
\hline CONSTANTE & $-0,319$ & 0,2830 & $-2,65$ & \\
\hline TCAM & $-0,041$ & 0,0686 & $-2,42$ & 1,25 \\
\hline TESP & $-0,0512$ & 0,0565 & $-2,26$ & 1,37 \\
\hline TVEH & $-0,0297$ & 0,0416 & $-2,51$ & 1 \\
\hline COSTO & $-0,00147$ & 0,0026 & $-1,66$ & 19,83 \\
\hline TRANSBORDO & $-0,378$ & 0,2352 & $-1,75$ & 7,35 \\
\hline Muestra & 568 & & & \\
\cline { 1 - 4 }
\end{tabular}

Fuente: Elaboración Propia

TABLA XIV.

ESCENARIO 3 ESTRATO 3 Y 4 EVALUADO

\begin{tabular}{l|c|c|c|c|c|}
\hline ESTRATO $3 Y 4$ & \multicolumn{3}{l|}{} \\
\hline VARIABLE & PARAMETRO & ERROR ESTANDAR & T-TEST & FP Y VST \\
\hline CONSTANTE & $-1,083$ & 0,416 & $-2,16$ & \\
\hline TCAM & $-0,113$ & 0,0473 & $-2,03$ & 1,89 \\
\hline TESP & $-0,0871$ & 0,0446 & $-1,96$ & 1,56 \\
\hline TVEH & $-0,0507$ & 0,0387 & $-2,1$ & 1 \\
\hline COSTO & $-0,000891$ & 0,0021 & $-2,36$ & 43,93 \\
\hline TRANSBORDO & $-0,385$ & 0,2132 & $-2,28$ & 5,16 \\
\hline Muestra & 876 & & & \\
\cline { 1 - 4 }
\end{tabular}

Fuente: Elaboración Propia

TABLA XV.

ESCENARIO 3 ESTRATO 5 EVALUADO

\begin{tabular}{|l|c|c|c|c|}
\hline ESTRATO 5 & \multicolumn{2}{|l|}{} & \\
\hline VARIABLE & PARAMETRO & ERROR ESTANDAR & T-TEST & FPYVST \\
\hline CONSTANTE & $-2,16$ & 2,631 & $-2,46$ & \\
\hline TCAM & $-0,115$ & 0,305 & $-2,53$ & 2,31 \\
\hline TESP & $-0,087$ & 0,287 & $-2,62$ & 1,95 \\
\hline TVEH & $-0,0362$ & 0,259 & $-2,78$ & 1 \\
\hline COSTO & $-0,00051$ & 0,01 & $-1,47$ & 63,16 \\
\hline TRANSBORDO & $-0,221$ & 0,986 & $-2,51$ & 3,12 \\
\hline Muestra & 100 & & & \multicolumn{1}{|l}{} \\
\cline { 1 - 3 } & & & &
\end{tabular}

Fuente: Elaboración Propia

$\mathrm{Al}$ apreciar los rangos de Test $-\mathrm{t}$ se puede afirmar que este escenario corresponde al modelo que mejor se ajusta a los datos ya que superaron el valor de 1.96. Se puede concluir finalmente que los valores aceptados de acuerdo al modelo son los siguientes:

\section{CONCLUSIONES}

Este es el primer modelo planteado en Bucaramanga y su Área Metropolitana en el que se intenta evaluar el factor tiempo pero teniendo en consideración el estrato al cual pertenece la persona encuestada.

Se observa la necesidad de un sistema correcto de planeación para las diferentes rutas, donde se integren todas las rutas actuales y así procurar evitar demoras en las mismas y mayor aceptación al sistema de transporte.

En el desarrollo del presente documento se observó que el modelo es muy sensible a la calidad de los datos tomados en campo.

Los datos obtenidos para el valor subjetivo del tiempo son los siguientes:

Como se aprecia donde se observa un mayor costo del valor subjetivo del tiempo se encuentra en el estrato 5 .

\section{5 . REFERENCIAS}

[1] J. D. D. Ortúzar, Modelos Econométricos De Elección Discreta, Santiago De Chile: Universidad Católica De Chile, 2000.

[2] J. D. D. Ortúzar, Modelos de demanda de transporte, México D.F.: Ediciones Universidad Católica de Chile - Alfaomega, 2000.

[3] C. Caliper, Travel demand modeling with transcad 4.8, Caliper: Caliper Corporation, 2005, p. 593.

[4] V. F. S. Ardila, Estimación de los factores de penalización del tiempo en la función de costo generalizado a bordo del sistema masivo transmilenio, Bogoá: Universidad Nacional de Colombia, 2009, p. 249.

[5] Steer Davied Gleave \& Hague Consulting Group, Marco Teorico adaptado de Stated Preference Techniques - A Guide To Practice, Australia: Kluwer Academic Publishers, 1991.

[6] J D. D. Ortuzar and C. Román, "El problema de la modelación de demanda desde una perspectiva desagregada: el codo del transporte," vol. XXIX, 2003, pp. 149-171. 
[7] J. J. P. Sartori, "Diseño de encuestas de preferencias declaradas para la estimación del valor de los ahorros de tiempo y el pronóstico de la demanda de servicios de transporte urbano de pasajeros," salta, 2006.

[8] T. Litman, "Introduction to multi -modal transportation planning," Victoria Transport Policy Institute, p. 19, 2014.

[9] G. de Rus, J. Campos and G. Nombela, Economía del transporte, Universidad de las Palmas de Gran Canaria, 2002.

[10] R. Cal y Mayor and J. Cardenas, Ingeniería del transito fundamentos y aplicaciones, Mexico: Alfaomega, 2007.

[11] S. D. d. Planeación, «Impacto económico de la movilidad en el distrito capital,» Bogotá,, 2008.

[12] I. M. d. Transporte, «MODELOS MATEMATICOS PARA DISTRIBUCION DE VIAJES,» Secretarua de Comunicaciones y Transportes, Querétano, 1991.

[13] D. Hensher, «Stated Preference Analysis Of Travel Choices: The State Of Practice,» Kluwer Academic Publishers, pp. 107-133, 1994.

[14] M. Anillo Galeano, Desarrollo de modelos para estimar la ponderación de los atributos del nivel de servicio en la toma de decisiones entre alternativas de transporte, Barranquilla: Universidad del Norte, 2006.

[15] J. Quintana Valido, «El tiempo de viaje como variable competitiva en el diseño de tarifas de transporte,» vector plus, Vols. \%1 de \%2EneroJunio, pp. 83-88, 2010.

[16] O. A. Alfonso, Modelos de elección discreta en trasnportes con coeficientes aleatorios, coruña: universidad de la coruña, 2005.

[17] S. Montalva y H. Emilio, Análisis microeconómico de politicas para combatir la congestion vial, santiago de chile: universidad de chile, 2010.
[18] I. M. d. Transporte, V. Rivera, C. Rivera y G. Torres, «Estudio de la demanda de transporte,» Secretaria de Comunicaciones y Transportes, Querétaro, 2002.

[19] M. Bierlaire y M. Fetiarison, «Estimation Of Discrete Choice Models: Extending Biogeme,» $S$ Transpor, p. 21, 2009.

[20] L. Montero Mercadé, Modelos de captación, análisis e interpretación de datos, Catalunya: Universidad Politecnica de Catalunya, 2012.

[21] Ministerio de Transporte de Colombia, «Manual de cálculo de los indicadores de monitoreo y evaluación de proyectos de transporte urbano en colombia,» Bogota, 2009.

[22] J. C. Mendieta, Apuntes de Clase - Introducción a la economía del transporte la teoría de la demanda de transporte urbano, Bogotá: Universidad de Los Andes, 2008.

[23] J. de Cea, E. Fernández, A. Salgado y M. Fernández, Calibracion de funciones flujo-costo para un modelo de asignacion de transporte publico con restriccion de capacidad, Santiago de Chile: Universidad Catolica de Chile, 2009.

[24] I. M. d. Transporte y E. Moreno Quintero, «Métodos de elección discreta en la estimación de la demanda de transporte,» Secretaria de Comunicaciones y Transporte, Querétaro, 2011.

[25] G. Kocur, Guide To Forecasting Travel Demand With Direct Utility, Washington, D.C: Urban Mass Transportation Administration-US Department of Transportation, 1982.

[26] J. E. C. Maquilon, C. A. Gonzalez y J. Posada, «Variables psicológicas en la eleccion de un modo de transporte urbano: integración de modelos de elección discreta,» de $10^{\circ}$ congreso colombiano de ingeniería de tránsito y transporte, Medellín, 2010.

[27] A. Morales Fernandez, Modelación y simulación de una vía rápida con rampas usando autómatas celulares, México: Instituto Politecnico Nacional, 2010.

[28] F. Yu-qin, «Route Choice Model Considering Generalized Travel Cost Based on Game Theory,» Mathematical Problems in Engineering, vol. 1, $\mathrm{n}^{\mathrm{o}}$ 464038, p. 5, 2013. 
[29] B. Si, «Travel demand-based assignment model for multimodal and multiuser transportation system,» Journal of Applied Mathematics, vol. 1, no 592104, p. 22, 2012. 